\title{
Benign Cystic Mesothelioma Misdiagnosed as Peritoneal Carcinomatosis
}

\author{
Hyun Deok Shin Suk Bae Kim \\ Department of Internal Medicine, Dankook University College of Medicine, \\ Cheonan, Korea, Republic of
}

\section{Keywords}

Benign cystic mesothelioma · Peritoneal carcinomatosis · Ascites

\begin{abstract}
Benign cystic mesothelioma (BCM) is a rare benign disease that forms multicystic masses in the abdomen, pelvis, and retroperitoneum. It occurs predominantly in young to middle-aged women. The majority of cases were associated with a history of abdominal or pelvic operation, a history of endometriosis, and pelvic inflammatory disease. We present a unique case of $\mathrm{BCM}$ which is different to the previous cases. The patient was a 52 -year-old man showing features of peritoneal carcinomatosis accompanied by ascites on abdominal computed tomography scans. We herein report a case of BCM misdiagnosed with peritoneal carcinomatosis.

\section{Introduction}

Benign cystic mesothelioma (BCM) is a very rare disease characterized by proliferative lesions of the peritoneal mesothelial cells mainly in the pelvic or abdominal cavity. However, it can also develop in the pleura, pericardium, tunica vaginalis, and spermatic cord [1]. In the past, it has also been named multicystic peritoneal mesothelioma, multilocular cysts of the peritoneum, multicystic mesothelioma, and multicystic peritoneal inclusion cysts $[1,2]$. It 


\section{Case Reports in \\ Gastroenterology}

Case Rep Gastroenterol 2016;10:115-120

(c) 2016 The Author(s). Published by S. Karger AG, Basel www.karger.com/crg

Shin and Kim: Benign Cystic Mesothelioma Misdiagnosed as Peritoneal Carcinomatosis

was first described by Mennemeyer and Smith [3] in 1979 as a possible diagnosis in a case that resembled intra-abdominal cystic hygroma. Its etiology is still not definite and its pathogenesis, which is reactive or neoplastic change, is controversial. The natural course of BCM is not well known. However, its tendency to recur after partial resection is well reported. There is a case report of a patient who died from the disease 12 years after the diagnosis without treatment [4]. Therefore, the first-line treatment is complete resection whenever possible. Although intraperitoneal chemotherapy after resection is reported as treatment in a large series of patients with peritoneal dissemination of appendiceal malignancy, there still is controversy about chemotherapy [5]. BCM is often observed in women, particularly those in their childbearing age and with a history of endometriosis, pelvic inflammation, and abdominal surgery. The rate of BCM in men is $17 \%$ [4]. The disease is sometimes found in patients without any typical symptoms; however, in most cases, large masses are palpable in the abdomen and abdominal pain often accompanies the disease. The authors report the case of a BCM in a 52-year-old middle-aged male patient who showed a unique pattern in that he had ascites and had first been misdiagnosed with peritoneal carcinomatosis based on radiological examination.

\section{Case Presentation}

A 52-year-old man was admitted to our hospital due to ascites found during a medical checkup performed in a private clinic. At the time of admission, his health status was normal: blood pressure 139/86 $\mathrm{mm} \mathrm{Hg}$, pulse rate 73 beats/min, respiratory rate $20 / \mathrm{min}$, and temperature $36.5^{\circ} \mathrm{C}$. He had no apparent trace of the disease and was conscious. The breathing sound and the heart sound during the chest examination were normal. There was a little abdominal distension, but there seemed to be no increase in the abdominal circumference and no tenderness. In the course of the previous few months, he presented no change in weight or peripheral edema of the limbs. The peripheral blood examination showed normal findings, i.e. leukocytes $7,820 / \mathrm{mm}^{3}$, hemoglobins $14.8 \mathrm{~g} / \mathrm{dl}$, and platelets $220,000 / \mathrm{mm}^{3}$.

The biochemical test results were as follows: C-reactive protein (CRP) $0.08 \mathrm{mg} / \mathrm{dl}$, BUN $13.2 \mathrm{mg} / \mathrm{dl}$, creatinine $0.87 \mathrm{mg} / \mathrm{dl}$, AST $30 \mathrm{IU} / \mathrm{l}$, ALT $38 \mathrm{IU} / \mathrm{l}$, total bilirubin $0.56 \mathrm{mg} / \mathrm{dl}$, and albumin $4.3 \mathrm{~g} / \mathrm{dl}$. The tumor marker test showed normal findings, i.e. $\alpha$-fetoprotein 1.8 $\mathrm{ng} / \mathrm{ml}$, carcinoembryonic antigen (CEA) $2.0 \mathrm{ng} / \mathrm{ml}$, and carbohydrate antigen 19-9 (CA 199) $11.5 \mathrm{U} / \mathrm{ml}$. Abdominal computed tomography (CT) scans presented ascites of a moderate amount. The irregularly thickened peritoneum across the abdomen and the reticular membrane in the upper left abdomen formed a mass (fig. 1). Positron emission tomography of the whole body showed no increase in the $18 \mathrm{~F}$-fluorodeoxyglucose uptake. The paracentesis conducted after the hospitalization resulted in an erythrocyte count of $171 / \mathrm{mm}^{3}$, leukocyte level of $288 / \mathrm{mm}^{3}$ (lymphocytes 74\%), albumin level of $2.0 \mathrm{~g} / \mathrm{dl}$, protein level of $2.7 \mathrm{~g} / \mathrm{dl}$, and adenosine deaminase level of $9.5 \mathrm{IU} / \mathrm{l}$, and there was no MTB PCR and AFB stain. As the abdominal CT findings strongly suggested peritoneal cancer, diagnostic laparoscopy was conducted.

The laparoscopic examination showed several cystic masses in the paracolic gutter forming a large mass (fig. 2a). There was much yellowish ascites, and multiple nodules of a size of 2-3 mm were widely spread in the abdominal cavity (fig. 2b).There was adhesion between the large reticular membrane and the pelvic wall. Biopsy was performed providing tissue of the size of $2 \times 1.5 \mathrm{~mm}$ from the reticular membrane. The biopsy result showed several cysts of various sizes, which were surrounded by flat cubic epithelial cells (fig. 3a). Im- 
munohistochemical staining was performed. As the biopsy did not stain for CD31 but strongly stained for calretinin and D2-40, the patient was diagnosed with BCM (fig. 3b). The patient was informed about the possible progression to malignancy and was recommended to have radical excision performed, but he preferred to wait since the symptoms were not yet apparent. We carried out an abdominal CT examination 2 months later. As there was no change in the lesion and there still was ascites, we recommended surgical treatment. However, he kept refusing it, and thus, we decided to continue monitoring the development.

\section{Discussion}

Although BCM was first referred to by Plaut [6] in 1928, it was suggested to have its origin in the mesothelial cells due to the observations made by Mennemeyer and Smith [3] using electron microscopy. Afterwards the disease could be diagnosed as soon as it had been distinguished from other diseases. After the introduction of immunohistochemistry, BCM was diagnosed when it was stained both for calretinin and D2-49 at the same time.

$\mathrm{BCM}$ is known to be found in women in their childbearing age. A report on the analysis of 37 patients with BCM showed that $83.8 \%$ of the patients were female and their average age was 38 years. As the disease is found in $37.8 \%$ of subjects in their 50 s, in $5.3 \%$ of subjects in their $60 \mathrm{~s}$ and in $10.8 \%$ of subjects in their $70 \mathrm{~s}$, as well as in infants, it is suggested that BCM may occur in patients at all ages. It is known to be more frequent in patients with a history of endometriosis, pelvic inflammation, and abdominal surgical treatment, particularly in patients having had hysterectomy and cesarean section [4].

$\mathrm{BCM}$ is a tumor originated from mesothelial cells in the peritoneum, pleura and pericardium. It can also appear in the groin and scrotum, which is the extension of the peritoneum. In the case of BCM in the groin and scrotum, the patients can detect palpable masses by themselves and therefore present themselves to the hospital earlier [1]. On the other hand, $\mathrm{BCM}$ in the abdominal or pelvic cavity is not easy to be found unless the mass is big. BCM in our case is also not easy to be detected because it is rare in a middle-aged man, and there were no specific symptoms such as palpable mass or abdominal distension. Worldwide, only 5 cases of BCM accompanied by ascites have been described so far [7]. Paracentesis results showed that his serum-ascites albumin gradient (SAAG) was $2.3 \mathrm{~g} / \mathrm{dl}$ and the protein level was $2.7 \mathrm{~g} / \mathrm{dl}$, showing that the disease may be caused by cardiac ascites. Thus, when more data about BCM with ascites are available in the future, the specific features of ascites in BCM could be analyzed.

BCM can be classified into three types depending on its shape: (1) the solitary type when the boundary is clear, (2) the localized type when the multiple masses are found in the localized part of the abdominal membrane, and (3) the diffuse type when the masses are widely spread across the abdominal membrane [4]. Surgical removal is the best treatment. To prevent possible recurrence and transformation to the malignancy, it is recommended to cut out a wide enough area whenever possible [8]. Worldwide, there were only 2 cases where BCM developed into the malignancy: a baby at the age of 6 months and a female at the age of 36 years $[9,10]$. Accordingly, as the rate of transformation to the malignancy is very low, reexcision is often performed even for local recurrence. In a few studies, there have been attempts to use heated intraperitoneal chemotherapy in the abdominal cavity, but this still is at an experimental stage [11]. 


\section{Conclusion}

$\mathrm{BCM}$ is a very rare disease, which is often observed in women in their childbearing age. We presented a male patient with BCM of the diffuse type accompanied by ascites and who had first been misdiagnosed with peritoneal carcinomatosis.

\section{Statement of Ethics}

The published research is compliant with the guidelines for human studies and animal welfare regulations.

\section{Disclosure Statement}

The authors have no conflicts of interest.

\section{References}

1 Ball NJ, Urbanski SJ, Green FH, Kieser T: Pleural multicystic mesothelial proliferation. The so-called multicystic mesothelioma. Am J Surg Pathol 1990;14:375-378.

-2 O'Connor DB, Beddy D, Aremu MA: Benign cystic mesothelioma of the appendix presenting in a woman: a case report. J Med Case Rep 2010;4:394.

3 Mennemeyer R, Smith M: Multicystic, peritoneal mesothelioma: a case report with electron microscopy of a case mimicking intra-abdominal cystic hygroma (lymphangioma). Cancer 1979;44:692-698.

4 Weiss SW, Tavassoli FA: Multicystic mesothelioma. An analysis of pathologic findings and biologic behavior in 37 cases. Am J Surg Pathol 1988;12:737-746.

5 Sugarbaker PH, Chang D: Results of treatment of 385 patients with peritoneal surface spread of appendiceal malignancy. Ann Surg Oncol 1999;6:727-731.

$6 \quad$ Plaut A: Multiple peritoneal cysts and their histogenesis. Arch Pathol 1928;5:754-758.

7 Manuc M, Lamatic C, Pop C, Dobrea C, Becheanu G, Grasu M, Iosif D, Diculescu M: Multicystic mesothelioma - a rare case of ascites: case report. Rev Med Chir Soc Med Nat Iasi 2007;111:895-900.

-8 Safioleas MC, Constantinos K, Michael S, Konstantinos G, Constantinos S, Alkiviadis K: Benign multicystic peritoneal mesothelioma: a case report and review of the literature. World J Gastroenterol 2006;12:5739-5742.

-9 DeStephano DB, Wesley JR, Heidelberger KP, Hutchinson RJ, Blane CE, Coran AG: Primitive cystic hepatic neoplasm of infancy with mesothelial differentiation: report of a case. Pediatr Pathol 1985;4:291-302.

-10 González-Moreno S, Yan H, Alcorn KW, Sugarbaker PH: Malignant transformation of 'benign' cystic mesothelioma of the peritoneum. J Surg Oncol 2002;79:243-251.

-11 Park BJ, Alexander HR, Libutti SK, Wu P, Royalty D, Kranda KC, Bartlett DL: Treatment of primary peritoneal mesothelioma by continuous hyperthermic peritoneal perfusion (CHPP). Ann Surg Oncol 1999;6:582-590. 


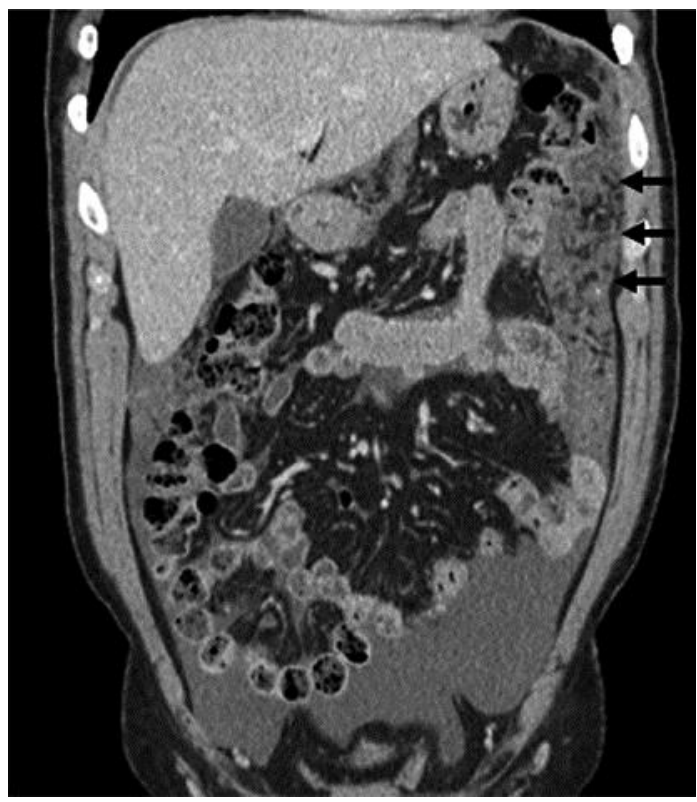

Fig. 1. Abdominal CT scan showing a moderate amount of ascites and diffuse peritoneal infiltration with omental cake formation (arrows).
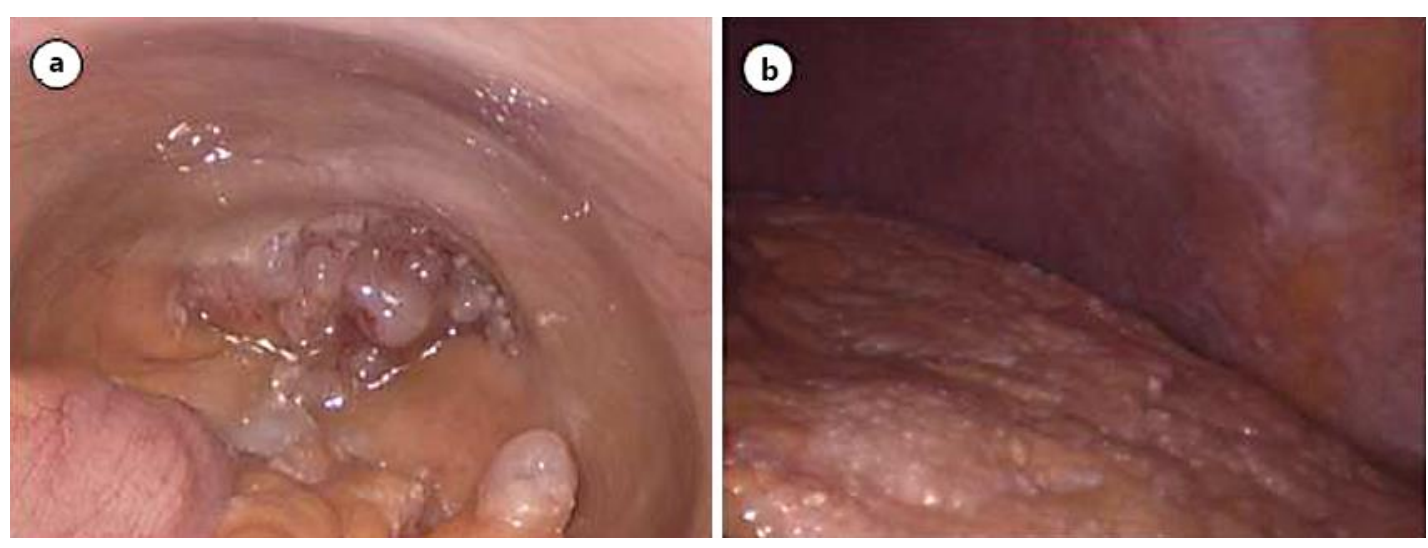

Fig. 2. Laparoscopic findings. a Variable-sized multicystic masses are seen in front of the paracolic gutter space. b Numerous 2- to 3-mm-sized nodular lesions that seemed like peritoneal seeding masses are observed in the peritoneal cavity. 


\section{Case Reports in Gastroenterology
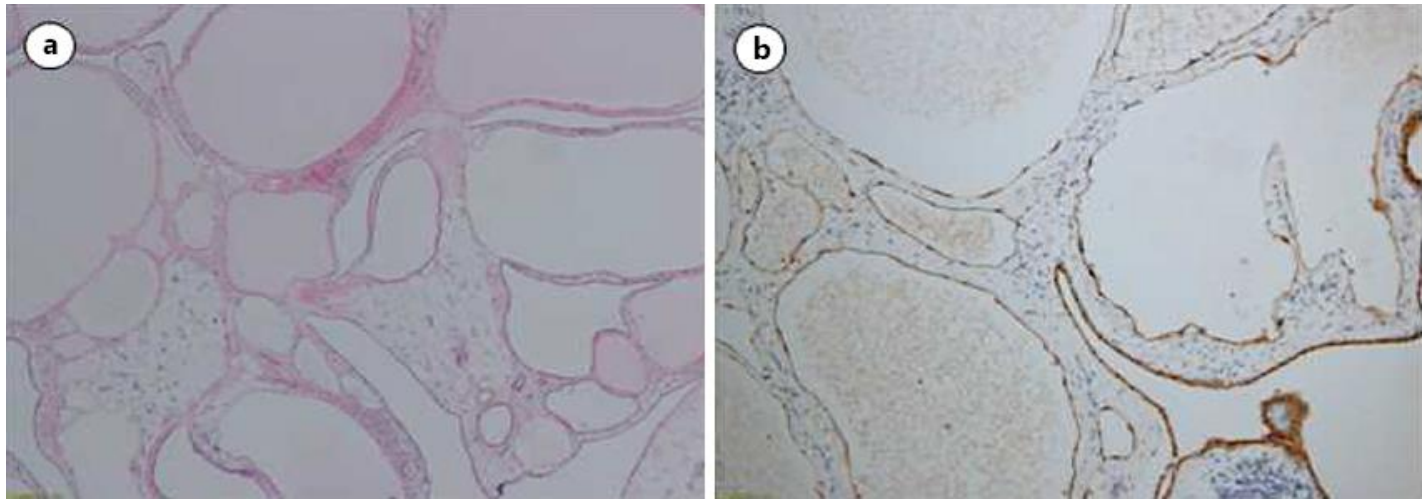

Fig. 3. Microscopic findings. a H\&E staining $(\times 100)$ reveals multiple variable-sized cysts lined with flattened epithelial cells. b Calretinin immunohistochemical staining $(\times 100)$ reveals a positive reaction. 